\title{
Characterization of codon usage pattern and evolutionary changes of Human Enterovirus 71 in China
}

\author{
Meng Wang ( $\sim$ nanadt@126.com ) \\ Nanfang Hospital \\ Li Chen \\ Nanfang Hospital \\ Penghui Sun \\ Nanfang Hospital \\ Wangjie Jin \\ Nanfang Hospital \\ Yanjiang Han \\ Nanfang Hospital \\ Shun Huang \\ Nanfang Hospital
}

\section{Research Article}

Keywords: Enterovirus 71, Codon usage, Correspondence analysis, phylogenetic trees

Posted Date: March 23rd, 2022

DOl: https://doi.org/10.21203/rs.3.rs-1406591/v2

License: (9) (i) This work is licensed under a Creative Commons Attribution 4.0 International License. Read Full License 


\section{Abstract}

Background: Enterovirus 71 (EV71) is a common etiological agent of hand, foot and mouth disease (HFMD) in children, which caused large outbreaks in mainland China in 2008. However, the genetic information of EV71 strains circulating in China is limited.

Methods: Here, we performed a systematic evolutionary and codon usage bias analysis of EV71.

Result: The result shows that the overall extent of codon usage bias in EV71 is low (ENc=56.696), and natural selection, mutation pressure, nucleotide compositions and host shape the codon usage patterns of EV71, with natural selection being more important than the others. We also found EV71 breaking out in large scale in 2008, the codon usage pattern of the virus isolated from China has change a lot compare the previous isolates, and after 2008, three different codon usage patterns were popularized in China.

Conclusion: In the end, we've confirmed that C2 isolates has outbreak in China before 2000 through codon usage analysis for the first time. In conclusion, our results provide useful insights into the evolutionary relationships of EV 71.

\section{Background}

Human Enteroviruses is single-stranded, positive-sense RNA viruses in the Picornaviridae family and has been classified into four species including over 100 serotypes. One of the most important serotypes was Enterovirus 71 (EV71) which is the major causative agent of hand-foot-and-mouth disease (HFMD) particularly aiming young children and infants, and often occurs in clusters or outbreaks worldwide [1, 2]. EV71 was first discovered in North America approximately one half century ago and has been found in many parts of the world. over the past decades, EV71 outbreaks in the Asia-Pacific region have affected millions of children, and caused thousands of deaths [3]. EV71 infection had been widely prevalent in China since 2007. For example, HFMD pandemics in Shandong Guangdong, Anhui Province, in 2008 resulted in tens of thousands of childhood infections and even dozens of deaths $[4,5]$.

Analysis of synonymous codon usage can provide the information on the mechanisms of synonymous codon-biased usage, the selection of appropriate host expression systems, the design of degenerate primers, gene prediction from genomic sequence[6, 7], and protein functional classification[8, 9]. Nonetheless, there are many reports on recombination between different genotypes of EV71 [10-12], suggesting that EV71 has high variability and recombination ability in China, which may lead to make a difference in codon usage. So far, little information about synonymous codon usage pattern of EV71 genome has been acquired to date. To assess the evolutionary adaptation of the virus to its hosts, we performed detailed analyses of codon usage and traditional phylogeny of EV71 based on 114 available complete open reading frames (ORF).In particular, 99 Chinese isolates of them was analyzed in detail. These studies might provide a novel perspective on the genetic divergence of EV71 and explore potential factors shaping its codon usage patterns in China. 


\section{Materials And Methods}

\section{Sequence data and phylogenetic analysis}

114 ORFs of EV71 were obtained from GenBank (https://www.ncbi.nlm.nih.gov/genbank/). The detailed information of the sequences, including accession number, country and year of isolation, are listed in supplementary materials (Table S1). in addition, 99 of them were isolated from China. Sequences were aligned by muscle in MEGA X[13].The Maximum Likehood (ML) tree was reconstructed based on the Tamura-Nei model implemented in MEGA X with the bootstrap value set at 2,500.

\section{The actual and predicted values of the effective number of codon (ENc)}

The ENc is used to measure the degree of departure from the equal use of synonymous codons of coding regions of EV71. The values of the effective number of codon (ENC) range from 20 to 61. In an extremely biased gene where only one codon is used for each amino acid, this value would be 20 ; if all codons are used equally, it would be 61; and if the value of ENc is greater than 40 , the codon usage bias was regarded as low. The values of ENc were obtained by EMBOSS CHIPS program.

The ENc- $(\mathrm{C}+\mathrm{G}) 3 \%$ plot [ENc plotted against $(\mathrm{G}+\mathrm{C}) 3 \%$ ] was used as a part of general strategy to investigate patterns of synonymous codon usage. Genes, whose codon choice is constrained only by a mutation bias, will lie on or just below the curve of the predicted values.

The predicted values of ENc expected were calculated as

$$
\text { ENc expected }=2+S+\frac{29}{S^{2}+(1-S)^{2}}
$$

where s represents the given $(\mathrm{G}+\mathrm{C}) 3 \%$ value [14].

\section{The calculation of the relative synonymous codon usage (RSCU)}

In order to minimize the effect of amino acid composition on codon usage, each ORF of EV71 is represented as a 59-dimensional vector. Each dimension corresponds to the RSCU value of one sense codon excluding Met, Trp and three stop codons. The RSCU values of codons in each ORF of EV71 were calculated according to the formula of previous reports.

$$
\operatorname{RSCU}=\frac{\mathrm{g}_{i j}}{\sum_{j}^{\mathrm{n}^{i} \mathrm{~g}_{i j}}} \mathrm{n}_{i}
$$

Where gij is the observed number of the ith codon for jth amino acid which has ni type of synonymous codons. The codon with RSCU value more than 1.0 has positive codon usage bias, while the value $<1.0$ 
has relative negative codon usage bias. When RSCU value is equal to 1.0, it means that this codon is chosen equally and randomly[15].

\section{Codon adaptation index (CAI)}

To reveal the adaptability of the EV71 gene to the selected hosts, the CAI were calculated using the CAIcal SERVER (http://genomes.urv.cat/CAlcal/RCDI/)[16]. The RSCU of the host (homo sapiens) was obtained from the Codon Usage Database (http://www.kazusa.or.jp/codon/)[17]. The CAI value ranges from 0 to 1.0.The higher the CAI value is, the better the virus adapted to its host[18]. Additionally, the significance regarding the respective clades and hosts were tested in variance analysis with double factor analysis without repetition.

\section{Statistical analysis}

Principal component analysis (PCA) was carried out to analyze the major trend in codon usage pattern in different genomes of EV71 (excluding non-coding regions). It is a statistical method that performs linear mapping to extract optimal features from an input distribution in the mean squared error sense and can be used by self-organizing neural networks to form unsupervised neural preprocessing modules for classification problems[14].

Correlation analysis was used to identify the relationship between codon usage bias and synonymous codon usage patterns of EV71. This analysis was implemented based on the Pearson Correlation analysis. All statistical analyses were carried out using the statistical analysis software SPSS Version 17.0 .

\section{Results}

\section{Evolution analysis and principal factor analysis}

To explore the phylogeny among the 99 strains of EV71 extracted from China, we constructed the phylogenetic trees using ML methods based on ORF of EV71. As shown in Fig. 1, all viruses are divided into three groups (C4, B5, C2), and that including the strains extracted from China.

Principal component analysis (PCA) is frequently used to study the major trend in codon usage pattern among EV71 samples. In the splattering chart of Fig. 2, all 114 strains of EV71 are divided into three groups. In addition, all strains extracted from China before 2008, except those extracted from Taiwan in 1998, are clearly distributed on the right of $X$ axis.

\section{Correlation analysis of ENc and $(C+G) 3 \%$}

As shown in Fig. 3, all strains are distributed below expectation curve, which proves that mutation pressure might play a more important role rather than natural selection in the evolution process of EV71 
virus. In addition, it is clear that strains of $\mathrm{C} 4$ are distributed on the left of the top, closer to the expectation line.

\section{Further analysis of each group}

As shown in Fig. 4A, B and C, B5 is clearly higher than the other two groups in CAl value, but lower in ENc value. Meanwhile, the linear regression coefficient of $B 5$ is the highest(Fig. $5 A, B$ and $C$ and Table 1)which revealed that $\mathrm{B} 5$ was more suitable for the host at codon usage pattern. In addition, no matter CAl, ENc or $(C+G) 3 \%$, the value of $C 2$ was all dispersive, resulting in large fluctuation. Meanwhile, as Fig. $6 A$, $B$ and $C$ shows, the $C A l, E N c$ or $(C+G) 3 \%$ values do not vary with time, except for $(C+G) 3 \%$ of $C 2$. Secondly, $(C+$ $\mathrm{G}) 3 \%$ value of $\mathrm{C} 2$ appeared for the first time in 2009 that vary greatly in codon usage pattern. As discovered in further studies (Fig.S1), the 6 strains of C2 virus are significantly different from other strains within the same group in terms of usage of codons such as AGA, CUU, CCU, UUG, AUU, CCA, CCU, UCA, ACC and UAC.

Table 1

The correlation analysis of those three groups (C4, B5, C2) and their hosts (Homo sapiens).

\begin{tabular}{|c|c|c|c|c|}
\hline & C4 & B5 & C2 & Homo sapiens \\
\hline \multirow[t]{2}{*}{ C4 } & & $r=0.875^{\star \star}$ & $r=0.919 * \star$ & $r=0.446^{\star \star}$ \\
\hline & & $p=0.000$ & $p=0.000$ & $p=0.000$ \\
\hline \multirow[t]{2}{*}{ B5 } & $r=0.875^{\star \star}$ & & $r=0.897 * \star$ & $r=0.542^{\star \star}$ \\
\hline & $p=0.000$ & & $p=0.000$ & $p=0.000$ \\
\hline \multirow[t]{2}{*}{ C2 } & $r=0.919 * \star$ & $r=0.897 \star \star$ & & $r=0.508^{\star \star}$ \\
\hline & $p=0.000$ & $p=0.000$ & & $p=0.000$ \\
\hline \multirow[t]{2}{*}{ Homo sapiens } & $r=0.479 \star \star$ & $r=0.542^{\star \star}$ & $r=0.508^{* *}$ & \\
\hline & $p=0.000$ & $p=0.000$ & $p=0.000$ & \\
\hline
\end{tabular}

\section{Correlation analysis}

The codon usage pattern of C4, B5 and C2 are highly correlated to their hosts, indicating that natural selection is playing a partial role in the evolution of the virus (Table 1). The highly significant correlation of codon usage pattern was found between the three groups. But a closer research revealed some codons of the three groups are clearly different as shown in line chart (Fig.S2), such as GCA, AGA, AGG, CGA, CGC, CGG, AAC, GGG, GGU, CUC, CUU, UUC, CCU, UCG and ACA. Compared with the other two groups, C2 is very different in terms of usage of GCA, CGG, AAC, AAU, GAU, CUU, UUU, UCG, ACA and GUG. 


\section{Discussion}

The codon usage analysis is the most essential feature that plays a major role in biological evolution. In this research, a series of analyses on ORF of 114 EV71strains was conducted. Firstly, nucleotide A was highly frequently presented in the genome ORF of EV71, on the contrary, which preferred $T$ at the third position of codon (Table S1). Secondly, the relatively low ENc values $(E N c=56.696>40$ in average) of all EV71 strains indicated low bias. In comparison with ENc value of other known RNA virus, such as polioviruses (HPV), hepadnaviruses, human immunodeficiency virus (HIV), Zika virus, West Nile virus $(E N C=53.754,44.45,51.9,52.72,53.81$ respectively) [19-22], a widely recognized explanation was that low bias was optimal for intracellular replication and immune evasion. Finally, it was discovered in this research that CAI values of all EV71 strains were relatively high, which was optimal for adaptation to the host and intracellular translation.

Analyses on the PCA and evolution tree reveals that the EV71 viruses that were popular in China before 2008 mainly belonged to C4 serotype, so it was more stable at that time. However, after 2008, a huge fluctuation on the codon usage pattern of virus was found, which is consistent with large scale outbreaks in Anhui, Guangxi, Jiangxi and other province includes 2008 years and later. This suggests that due to the population surge during the grant outbreak, EV71 faced selective and mutational pressure that led to diversity of its codon usage pattern. The diversity of codon usage pattern has changed some of the genetic characteristics of the EV71 virus, which will bring new pressure to the prevention and treatment of the virus in the future. And all of the above are similar to our previous researches [23]. On the other hand, it is discovered in principal factor analysis that the two isolates extracted from Taiwan in 1998 were greatly different from those in mainland China during the same period, which belonged to $\mathrm{C} 2$ serotype as verified by evolution tree analysis. It is proved for the first time through codon usage analysis that there outbreak of C2 isolates in China before 2000.

In this study, our analysis reveals that codon usage bias in EV71 is low, and mutation pressure is the main factor that affects codon usage variation in evolution of the virus. Other factors, including base composition, genotype, geography and host may also significantly influence codon usage bias. Our study aslo provides a basic understanding of the codon usage patterns of EV71 and the roles played by mutation pressure and natural selection. In addition, we studied the variation trend of codon usage pattern of EV71 in China, especially the great differences in codon usage pattern of the China isolates before and after 2008. But a more comprehensive analysis is needed to reveal more information about codon usage bias variation within EV71 viruses.

\section{Declarations}

\section{Conflict of interest}

We declare that we do not have any commercial or associative interest that represents a conflict of interest in connection with the work submitted. 


\section{Author contributions}

All authors contributed to producing the manuscript.

\section{References}

1. Sueoka, N (1988) Directional mutation pressure and neutral molecular evolution. Proc Natl Acad Sci U S A 85: 2653-7.

2. Li, B.Q, et al (2017) A Novel Enterovirus 71 (EV71) Virulence Determinant: The 69th Residue of 3C Protease Modulates Pathogenicity. Frontiers in Cellular and Infection Microbiology 7.Doi:10.3389/fcimb.2017.00026.

3. Zhang, Y.X, et al (2017) A highly conserved amino acid in VP1 regulates maturation of enterovirus 71. Plos Pathogens 13. D oi:org/10.1371/journal.ppat.1006625.

4. Zhu, Z, et al (2010) Retrospective seroepidemiology indicated that human enterovirus 71 and coxsackievirus A16 circulated wildly in central and southern China before large-scale outbreaks from 2008. Virology Journal 7.http://www.virologyj.com/content/7/1/300.

5. Guan, D.W, et al (2012) Population Dynamics and Genetic Diversity of C4 Strains of Human Enterovirus 71 in Mainland China, 1998-2010. Plos One 7. Doi:10.1371/journal.pone.0044386.

6. Chen, $\mathrm{L}$, et al (2013) Analysis of codon usage patterns in Taenia pisiformis through annotated transcriptome data. Biochemical and Biophysical Research Communications 430: 1344-1348.

7. Wang, B, et al (2021) Allosteric Activation of SARS-CoV-2 RNA-Dependent RNA Polymerase by Remdesivir Triphosphate and Other Phosphorylated Nucleotides. Mbio 12. Doi:10.1128/mBio.0142321.

8. Lin, Z, et al (2021) Evolution trace of SARS-CoV-2 from January 19 to March 12, 2020, in the United States. J Med Virol 93: p. 6595-6604.

9. Yahaya, R.S.R., et al (2021) Molecular strategies to increase keratinase production in heterologous expression systems for industrial applications. Applied Microbiology and Biotechnology 105: p. 3955-3969.

10. Sun, H, M. Gao, and D. Cui (2020) Molecular characteristics of the VP1 region of enterovirus 71 strains in China. Gut Pathog 12: p. 38.

11. Yu, F, et al (2020) Sub-genotype change and recombination of coxsackievirus A6s may be the cause of it being the predominant pathogen for HFMD in children in Beijing, as revealed by analysis of complete genome sequences. Int J Infect Dis 99: p. 156-162.

12. Liu, Z.W, et al (2019) Enterovirus 71 VP1 Protein Regulates Viral Replication in SH-SY5Y Cells via the mTOR Autophagy Signaling Pathway. Viruses,. 12(1).

13. Kumar, S, et al (2018) MEGA X: Molecular Evolutionary Genetics Analysis across Computing Platforms. Mol Biol Evol 35: p. 1547-1549.

14. Wang, M, et al (2011) Analysis of codon usage in Newcastle disease virus. Virus Genes 42: p. 245-53. 
15. Wang, $M$, et al (2011) Analysis of codon usage in type 1 and the new genotypes of duck hepatitis virus. Biosystems 106: p. 45-50.

16. He, W, et al (2019) Genetic analysis and evolutionary changes of Porcine circovirus 2. Mol Phylogenet Evol 139: p. 106520.

17. Je, M, H. Kim, and H.S. Son (2019) Analysis of the codon usage pattern of the RdRP gene of mycovirus infecting Aspergillus spp. Virol J 16: p. 10.

18. He, Z, H. Gan, and X (2019) Liang, Analysis of Synonymous Codon Usage Bias in Potato Virus M and Its Adaption to Hosts. Viruses11. D oi:10.3390/v11080752.

19. Wang, $\mathrm{M}$, et al (2011) Analysis of codon usage in bovine viral diarrhea virus. Archives of Virology 156: p. 153-160.

20. Deb, B, A. Uddin (2020) Chakraborty, Codon usage pattern and its influencing factors in different genomes of hepadnaviruses. Arch Virol 165: p. 557-570.

21. Phakaratsakul, S, et al (2018) Codon usage of HIV regulatory genes is not determined by nucleotide composition. Archives of Virology 163: p. 337-348.

22. Moratorio, G, et al (2013) A detailed comparative analysis on the overall codon usage patterns in West Nile virus. Infection Genetics and Evolution 14: 396-400.

23. Wang, M, et al (2020) Genetic and evolutionary analysis of enterovirus 71 base dinucleotide. VirusDis. Doi:10.1007/s13337-020-00569-z.

\section{Figures}




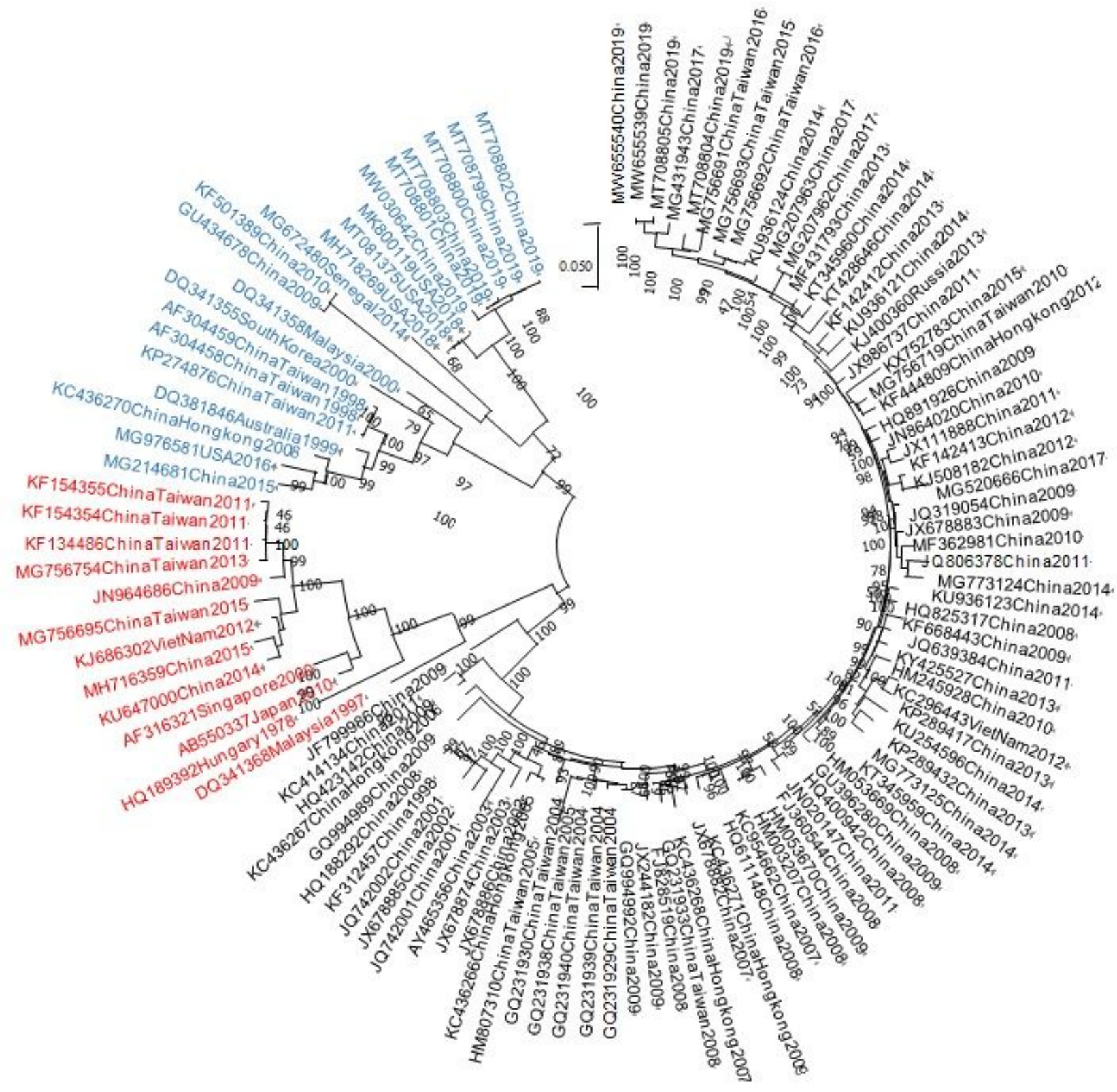

Figure 1

Phylogenetic tree representing relationship among the 114 strains of EV71. Each group symbolized by different color, such as group C2 was in blue『B5 was in red, C4 was in black. 


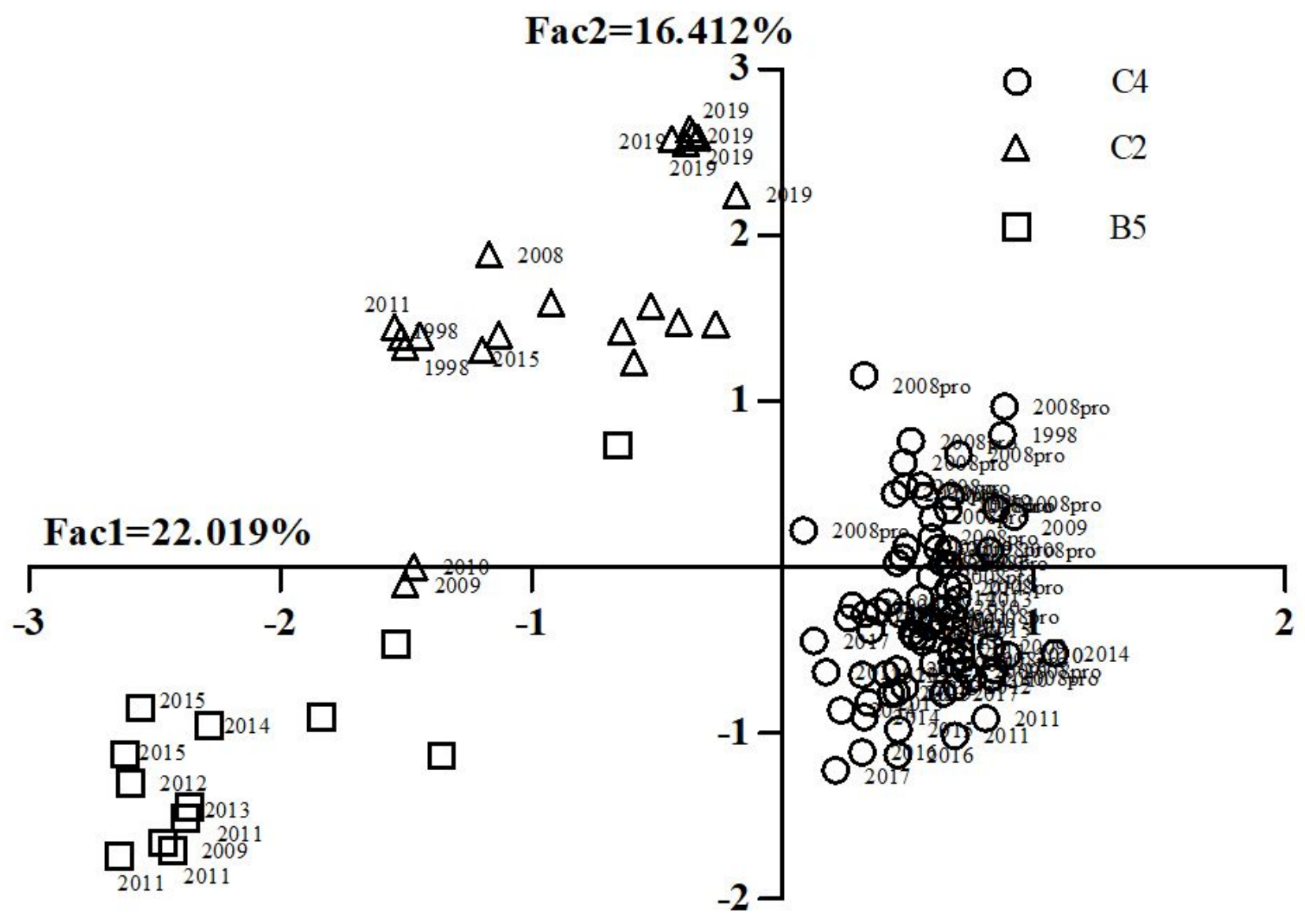

Figure 2

A plot of the values of the Fac1 (22.019\%) and the Fac2 (16.412\%) of 114 ORFs of EV 71 genomes in principle component analysis. 2008pro was stand for the EV 71 isolates including and before 2008, apart from the three isolates in 1998. 


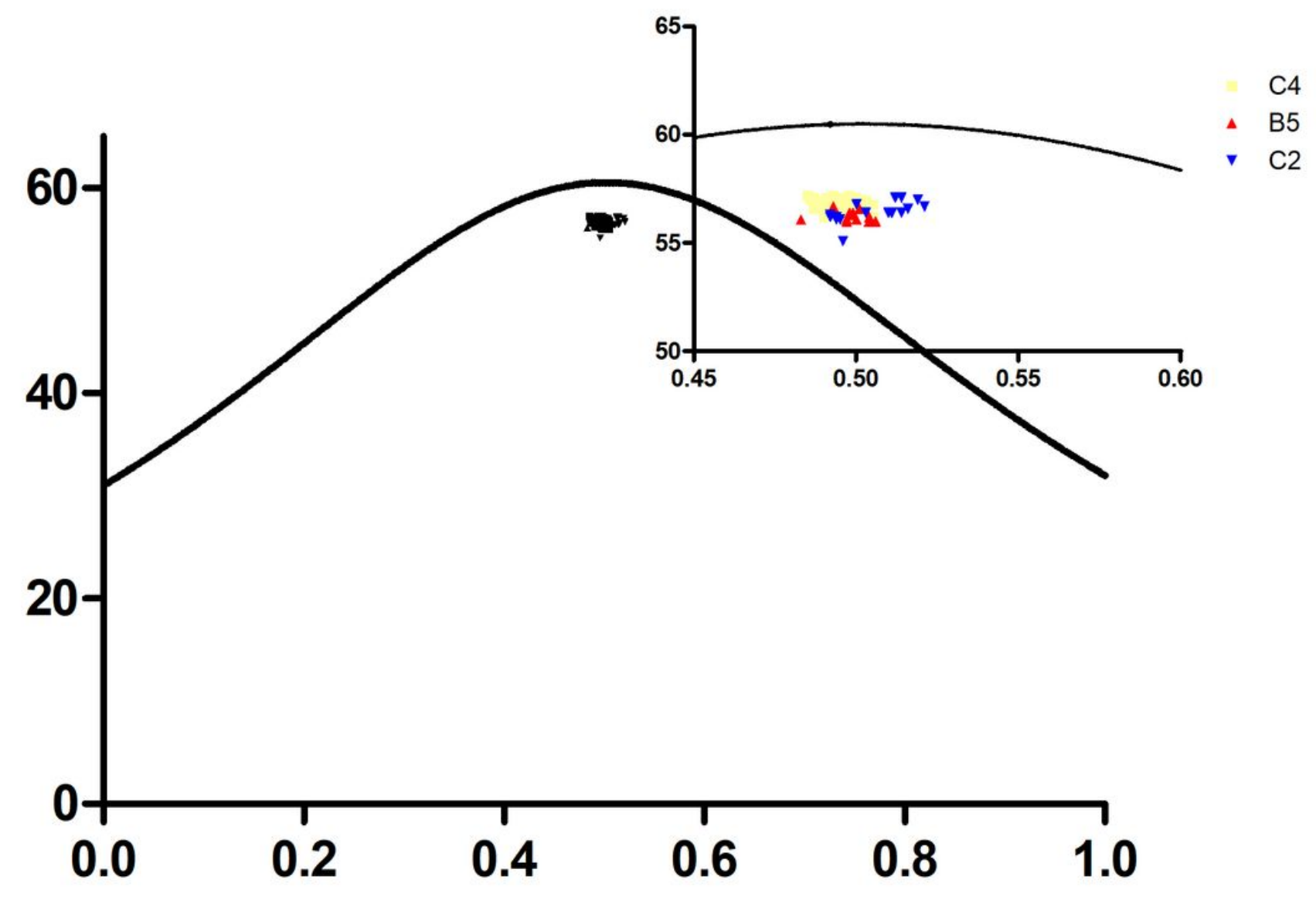

Figure 3

The ENc- $(C+G)_{3} \%$ plot Graphs showing the relationship between ENc and $(C+G) 3 \%$. The curve indicates the expected codon usage if GC compositional constraints alone account for codon usage bias.
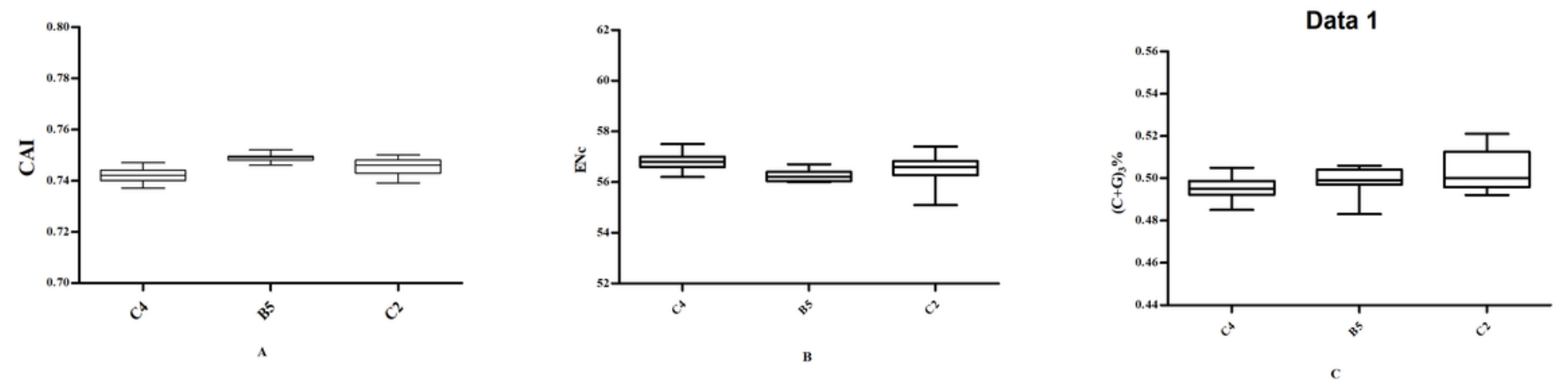

Figure 4 
CAl value of the three groups (C4, B5, C2); B:ENc value of the three groups; C: (C+G)3\% of the three groups. The data was presented as mean \pm SD.
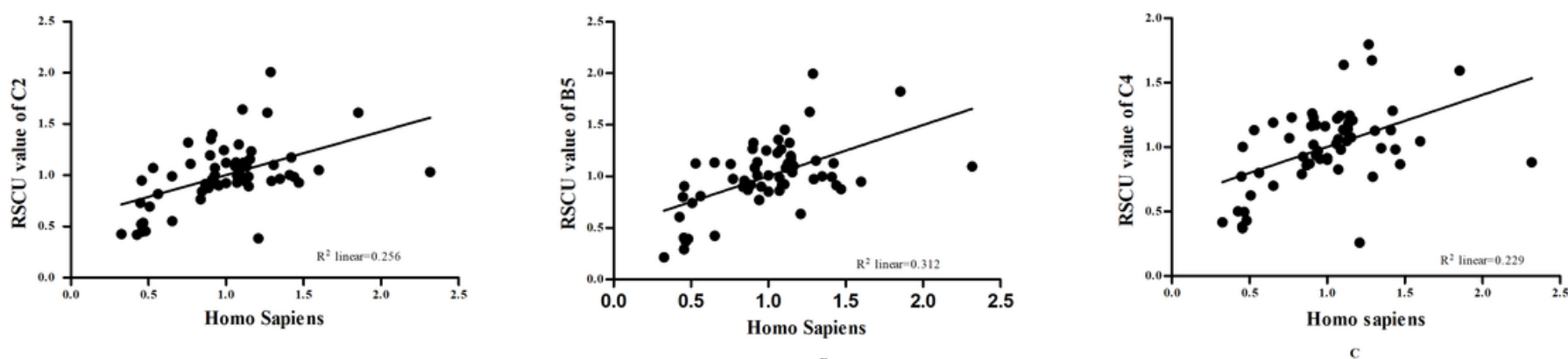

Figure 5

A, B and C: Correlation between the host (Homo sapiens) and the three groups (C4, B5, C2) based on the RSCU value, respectively.

Figure 6

A: CAl value of the three groups (C4, B5, C2) change with time; BQENc value of the three groups (C4, B5, C2) change with time; $\mathrm{CX}(\mathrm{C}+\mathrm{G}) 3 \%$ of the three groups $(\mathrm{C} 4, \mathrm{~B} 5, \mathrm{C2})$ change with time.

\section{Supplementary Files}

This is a list of supplementary files associated with this preprint. Click to download.

- Fig.S1.jpg

- Fig.S2.jpg

- TableS1.xlsx 